\title{
Dezentrale „Euthanasie“ in der Heil- und Pflegeanstalt Niedernhart. Untersuchung am Beispiel ausgewählter Krankenakten
}

\author{
Anna Kirchgatterer \\ Kerngebiet: Zeitgeschichte \\ eingereicht bei: Univ.-Prof. Mag. Dr. Dirk Rupnow \\ eingereicht im: SoSe 2019 \\ Rubrik: Bachelor-Arbeit (Lehramt)
}

\begin{abstract}
Wild "Euthanasia" in the care and nursing home Niedernhart. An analysis based on medical records

This paper deals with medical records from the Upper Austrian care and nursing home Niedernhart. Under the National Socialist regime, especially after "Aktion T4" was discontinued in August 1941, thousands of people were medically treated in this institution and many of them died. In line with the current state of research, this paper does not question that people were intentionally killed by the director of the institution, Dr. Lonauer, and some of his staff, but rather examines whether targeted killing of patients can be proven by scrutinizing medical records. Twelve of these documents are reviewed in detail.
\end{abstract}

\section{Einleitung}

Die Lern- und Gedenkstätte Hartheim ist vielen, vor allem in Oberösterreich, bekannt. An die dort während der "Aktion T4" des NS-Regimes ermordeten Menschen wird in einer Ausstellung erinnert. Die „Aktion T4", „die Tötung geistig und körperlich behinderter Menschen, war der erste systematisch geplante, staatlich durchgeführte Massenmord des NS-Regimes." Weniger bekannt ist hingegen, was nach dem Stopp dieses

1 Wolfgang Neugebauer, Die „Aktion T4“, in: Brigitte Kepplinger u. a. (Hrsg.), Tötungsanstalt Hartheim, Linz 2008, S. 17-34, hier S. 17. 
Programms passiert ist: An die in der Heil- und Pflegeanstalt Niedernhart bei Linz, dem heutigen Neuromed Campus, ermordeten Menschen erinnert keine dauerhafte Ausstellung. Trotzdem sind auch hier zahlreiche Menschen unter dem damaligen Direktor der Anstalt, Rudolf Lonauer, getötet worden. Mithilfe der Erstellung von Statistiken wurde versucht, sich an die durchgeführten anzunähern (vgl. Kapitel 2.2), einige Forschungsgruppen behandeln die Thematik aber auch mithilfe der Untersuchung von Krankenakten. ${ }^{2}$ Derartige Akten werden in dieser Bachelorarbeit ebenso, wenn auch in einem kleineren Rahmen, untersucht. Dabei sind gerade zur Heil- und Pflegeanstalt Niedernhart keine vergleichbaren Studien bekannt.

Die Forschungsfrage der vorliegenden Arbeit lautet: Kann in der Heil- und Pflegeanstalt Niedernhart anhand von Krankenakten dezentrale „Euthanasie“ zur Zeit des NSRegimes rekonstruiert werden? Dazu wird folgende These aufgestellt: Die Ermordung von Patient*innen durch Narkotika, Nahrungsentzug, mangelndes Heizen oder Vorenthaltung wichtiger Medikamente kann indirekt - beispielsweise durch nur sehr kurze Verweildauer in der Heil- und Pflegeanstalt, schnelle Gewichtsreduktion oder fehlende Verlaufseintragungen zu Krankheiten - vermutet werden.

Um diese These zu untersuchen, wird zu Beginn in einem kurzen Abriss dezentrale „Euthanasie" während des NS-Regimes näher dargestellt und dabei auf zwei für diese Arbeit relevante Studien Bezug genommen. Im Anschluss folgen detaillierte Ausführungen über die Situation in der Heil- und Pflegeanstalt Niedernhart. Dass durch den Leiter der Anstalt, Rudolf Lonauer, Patient*innen getötet wurden, ist in der Literatur nicht umstritten. Gerhart Marckhgott hat dazu unter anderem anhand der Altakten des heutigen Neuromed-Campus einige Statistiken erstellt, die kurz vorstellgestellt werden. Das folgende Kapitel ist schließlich der Kern dieser Arbeit: Dabei wird zunächst erörtert, unter welchen Aspekten das Korpus (bestehend aus zwölf Krankenakten von Patient*innen aus der Heil- und Pflegeanstalt Niedernhart, verstorben zwischen September 1941 und Mai 1943) untersucht wird. Die Quellen werden vorgestellt und die Ergebnisse erläutert. Die Anzahl der Krankenakten ist hinsichtlich der Forschungsfrage eine nur geringe, mit Hinweis auf den geforderten Umfang dieser Bachelorarbeit aber die einzig praktikable Möglichkeit. Eine Erklärung der Auswahl des Samples findet sich in Kapitel 3.2.

Wichtige Literatur für diese Arbeit ist unter anderem ein bereits etwas älterer Aufsatz von Gerhart Marckhgott: „'Euthanasie' in Oberdonau“, der sich über Statistiken an die Morde in Niedernhart annähert und auch in der Forschungsliteratur immer wieder zitiert wird. Daneben liefert ein Aufsatz von Brigitte Kepplinger, „Regionalisierter Krankenmord. Voraussetzungen und Strukturen der nationalsozialistischen Patiententö-

2 So zum Beispiel: Michael von Cranach u. a. (Hrsg.), Gedenkbuch für die Münchner Opfer der nationalsozialistischen „Euthanasie"-Morde, Göttingen 2018; Felicitas Söhner u. a., Nach der Aktion T4. „regionalisierte Euthanasie” in der Heil- und Pflegeanstalt Günzburg, in: Nervenarzt 88 (2017), Heft 9, S. 1065-1073; Gabriele Caprano-Diehl, Euthanasie-Verdacht in der Heil- und Pflegeanstalt Klingenmünster 1944-1946, Marburg 2012. Insgesamt gebe es, so Söhner, aber gerade zu dezentralisierter „Euthanasie“ in verschiedenen Teilen Deutschlands noch Lücken. Zu Österreich konnte leider keine vergleichbare Studie gefunden werden. In dieser Arbeit wird auf die ersten beiden hier genannten Werke Bezug genommen. 
tung außerhalb der zentral gesteuerten Programme", wichtige Informationen für die ersten beiden Kapitel. Ebenso interessant ist eine Monografie von Tom Matzek, „Das Mordschloss". ${ }^{\prime 3}$ ür die Auswertung der Krankenakten stützte sich die Arbeit vor allem auf Studien zu diesem Thema - von Michael Cranach u. a. "Gedenkbuch für die Münchner Opfer der nationalsozialistischen,Euthanasie'-Morde" und von Felicitas Söhner u. a. "Nach der Aktion T4., Regionalisierte Euthanasie' in der Heil- und Pflegeanstalt Günzburg".

Das Quellenkorpus für die vorliegende Bachelorarbeit stammt aus dem Archiv des Gedenkschlosses Hartheim, in dem die Krankenakten der Heil- und Pflegeanstalt Niedernhart aufbewahrt werden. Unterstützt wurde die Autorin bei der Quellenrecherche von Peter Eigelsberger, Mitarbeiter der Dokumentationsstelle Hartheim, der mit seinen Einschätzungen zu den Fällen beigetragen hat.

\section{Dezentrale „Euthanasie“}

\subsection{Ein Abriss}

Am 24. August 1941 wurde die "Aktion T4" eingestellt. ${ }^{5}$ Benannt wurde diese nach der Planungs- und Verwaltungsbehörde der "Euthanasie"-Morde, der Tiergartenstraße 4 in Berlin. Auf die zentral organisierte Ermordung von Patient*innen mit Schizophrenie, Epilepsie, seniler Demenz, Enzephalitis, Chorea Huntington oder anderen Diagnosen in dafür eingerichteten Häusern folgten die, teilweise vielleicht auch schon davor durchgeführten, dezentralen Anstaltsmorde. Eberhard Gabriel nennt hier zwei Verfahrensweisen: Zum einen wurde die Sterberate in den Anstalten durch Hunger und Vernachlässigung, zum anderen durch die direkte Tötung der Patient*innen mit Gift und elektrischen Strom erhöht. ${ }^{6}$ Oft wendeten die Verantwortlichen aber auch beides an: Die geschwächten Patient*innen wurden mit überdosierten Medikamentengaben getötet. Zu dieser Zeit gab es, anders als während der "Aktion T4", kein zentrales Begutachtungsverfahren mehr, über das Leben oder den „Lebenswert” entschieden nun Ärzt*innen und Pflegepersonal. Ein Fluchtversuch, Aufsässigkeit, Unsauberkeit oder Selbstbefriedigung konnten das Todesurteil für die Patient*innen sein.?

3 Brigitte Kepplinger, Regionalisierter Krankenmord. Voraussetzungen und Strukturen der nationalsozialistischen Patiententötung außerhalb der zentral gesteuerten Programme, in: Bertrand Perz u. a. (Hrsg.), Schlussbericht der Kommission zur Untersuchung der Vorgänge um den Anstaltsfriedhof des Psychiatrischen Krankenhauses in Hall in Tirol in den Jahren 1942 bis 1945, Innsbruck 2014, S. 49-82; Gerhart Marckhgott, Euthanasie in Oberdonau, in: Zeitgeschichte 21 (1994), Heft 5, S. 165-182; Tom Matzek, Das Mordschloss. Auf den Spuren von NS-Verbrechen in Schloss Hartheim, Wien 2003.

4 Söhner u. a., Nach der Aktion T4, S. 1065-1073; Sibylle von Tiedemann/Geritt Hohendorf, Methodik. Die Ermittlung der Opfer der dezentralen „Euthanasie“, in: Michael von Cranach u. a. (Hrsg.), Gedenkbuch für die Münchner Opfer der nationalsozialistischen „Euthanasie“-Morde, Göttingen 2018, S. 169-192.

5 Matzek, Das Mordschloss, S. 171.

6 Eberhard Gabriel, NS-Euthanasie in Österreich von 1938 bis 1945. Die Ereignisse - eine Rekapitulation, in: psychopraxis.neuropraxis 19 (2016), Heft 1, S. 21-24, hier S. 21-23.

$7 \quad$ Hans-Walter Schmuhl, Rassenhygiene, Nationalsozialismus, Euthanasie. Von der Verhütung zur Vernichtung „lebensunwerten Lebens“ 1890-1945 (Kritische Studien zur Geschichtswissenschaft 75), Göttingen 1987, S. 223. 
Das zentrale Büro in der Tiergartenstraße ging von einem vorübergehenden Stopp der „Aktion T4" aus und führte die Erfassung der Anstaltsinsassen trotzdem fort. ${ }^{8}$ Bei einer Konferenz in Pirna-Sonnenstein im November 1941 wurde, so Hans-Walter Schmuhl, den Ärzt*innen schließlich erklärt, dass Krankentötungen in Zukunft von zuverlässigen Ärzt*innen und Pfleger*innen durchgeführt werden sollten. Es handle sich dabei um Verfahren ohne Norm. ${ }^{9}$ Neugebauer schreibt dazu, dass es nicht klar sei, ob die Euthanasieaktionen tatsächlich aufgrund zentraler Anweisungen durchgeführt wurden. Die Zahl der Toten schätzt er auf jene Zahl an Menschen, die durch die "Aktion T4" zu Tode gekommen waren. ${ }^{10}$ Faulstich schätzt die Zahl der NS-Opfer in staatlichen Anstalten außerhalb der "Aktion T4" hingegen mit 5.000 geringer ein. ${ }^{11}$ Die Schätzung der Opferzahlen dezentraler „Euthanasie“ ist schwierig, festzuhalten ist aber: Obwohl auch während und nach dem Ersten Weltkrieg die Sterblichkeitsrate wegen Unterernährung hoch war, zeigt "doch ein Vergleich der Prozentzahlen [...] den verbrecherischen Eingriff: 1918 starben 15,5\% der Kranken in Eglfing, 1945 waren es 28,6\%".12 Im Folgenden werden zwei Studien zu dezentraler Euthanasie vorgestellt: Erstere befasst sich hauptsächlich mit den Vorgängen in der Pflegeanstalt Eglfing-Haar, sie ist für die Arbeit vor allem durch ihre Vorgehensweise in diesem Kontext interessant. In einer weiteren Studie wurden Krankenakten aus der Pflegeanstalt Günzburg untersucht (erschienen 2017 und 2018). Diese Untersuchung weist eine ähnliche Vorgehensweise auf, besticht aber durch die vorsichtigere Herangehensweise, welche für diese Arbeit als Vorbild gilt.

\subsection{Versuche zur Erfassung der Opfer dezentraler "Euthanasie"}

Sibylle von Tiedemann u. a. untersuchten in ihrer Forschungsarbeit, ob es sich bei den Verstorbenen mit Münchner Wohnsitz der Anstalt Eglfing-Haar um natürliche Tode oder um Morde handelte. Beurteilt wurden alle Krankenakten der Patient*innen, die zwischen 1. September 1939 und 31. Juli 1945 verstorben sind. Begründet wurde dieser Zeitraum mit den Nachwirkungen der Behandlung während der NS-Zeit. Die als „nicht natürlich" kategorisierten Tode wurden in das Gedenkbuch aufgenommen. ${ }^{13}$ Diese Untersuchung ist vor allem aufgrund der Kriterien zur Unterscheidung zwischen natürlichem und nicht-natürlichem Tod interessant.

Die Forschungsgruppe definiert dezentrale "Euthanasie" als:

„Tod infolge von Mangel an Nahrung, Kleidung, Heizung, medizinischer und pflegerischer Versorgung (strukturelle Vernachlässigung) ebenso wie der bewusste Verzicht auf pflegerische und medizinische Behandlungen, um einen

Winfried Süß, Der "Volkskörper" im Krieg. Gesundheitspolitik, Gesundheitsverhältnisse und Krankenmord im nationalsozialistischen Deutschland 1939-1945, München 2003, S. 314-315.

9 Schmuhl, Rassenhygiene, S. 220-222.

10 Wolfgang Neugebauer, NS-Euthanasieaktionen in Österreich. Ein Überblick, in: Bertrand Perz u. a. (Hrsg.), Schlussbericht der Kommission zur Untersuchung der Vorgänge um den Anstaltsfriedhof des Psychiatrischen Krankenhauses in Hall in Tirol in den Jahren 1942 bis 1945, Innsbruck 2014, S. 35-48, hier S. 43.

11 Heinz Faulstich, Hungersterben in der Psychiatrie. Mit einer Topographie der NS-Psychiatrie, Freiburg im Breisgau 1998, S. 582.

12 Schmuhl, Rassenhygiene, S. 222.

13 von Tiedemann/Hohendorf, Methodik, S. 172-173. 
bestimmten Patienten sterben zu lassen (individuelle Formen der Vernachlässigung), der systematische Nahrungsmittelentzug (Hungerkost) und die gezielte Tötung durch Medikamente."14

Anhand der Selektionskriterien der "Aktion T4" wurde dann versucht, die Todesursachen der Verstorbenen festzulegen. Kategorisiert wurden die Todesursachen in natürlichen Tod, Tod durch Nahrungsentzug, Tod durch Vernachlässigung, Tod durch überdosierte Medikamente und Tod in Therapie sowie Grenzfälle zwischen den Todesursachen. "Tod durch Nahrungsentzug" ließ sich aufgrund der Unterbringung der Patient*innen - in Eglfing gab es Hungerhäuser, in denen die Patient*innen fettlos ernährt wurden - leicht zuordnen. Außerdem sind in den untersuchten Akten meist Gewichtstabellen zu finden, die in jenen von Niedernhart fehlen. Zur Kategorie "Tod durch Vernachlässigung" zählen die Autor*innen Fälle, in denen aufgrund langer Krankheit von einer lebensrettenden Operation abgesehen wurde. Daneben wurden die 28 Opfer der Ruhrepidemie erfasst, da diese Zahl ohne den herrschenden Platzmangel und die fehlenden Isolationsmöglichkeiten geringer hätte sein können. Auch die Auswirkungen der schwierigen Versorgungslage wurden eingerechnet. „Tod durch überdosierte Medikamente" wird, so die Studienautor*innen, zum Beispiel durch erst spät niedergeschriebene Symptome festgestellt. So wurde zum Beispiel immer wieder eine Diskrepanz zwischen dem Aufscheinen der Symptome in den Akten und dem üblichen Krankheitsverlauf, beispielsweise bei Lungenerkrankungen, entdeckt. Hilfreich waren hier auch die Sektionsbefunde. Die vorletzte Kategorie wird verwendet, wenn ein „nicht intendierter Tod im Rahmen der Therapiemaßnahmen" eintritt. Darunter verstehen die Studienautor*innen den Tod durch eine dokumentierte medizinische Maßnahme wie Elektroschocktherapie oder Medikamente. ${ }^{15}$ Um die Todesursachen zu klären, orientierten sich die Verantwortlichen zunächst an den Selektionskriterien der "Aktion T4" Arbeitsfähigkeit, Pflegeaufwand, Kontakte zu Angehörigen, unerwünschtes, störendes Verhalten in der Anstalt - und legten so Kriterien zur Einordnung von natürlichem und nicht natürlichem Tod der Patient*innen fest. Zu diesen zählen Verhaltensbeschreibungen, Pflegeaufwand, familiäre Kontakte, Sprache in den Krankenakten, Gewicht und Verlaufseintragungen der Krankengeschichte.

Wurde das Verhalten in den Akten als "störend" oder gar "gefährlich" beschrieben, ist dies, so die Studienautor*innen, ein Indiz für einen „nicht natürlichen“ Tod, ebenso, wenn Patient*innen als „unrein" oder mit hohem „Überwachungsaufwand" beschrieben wurden. Familiäre Kontakte sind letztendlich nur schwer nachzuvollziehen, selbst wenn Briefe oder Eintragungen zu Besuchen erhalten sind. Abwertende oder entwürdigende Sprache sei ein Indiz für einen unnatürlichen Tod. ZurVerlaufseintragung in der Krankengeschichte wird festgehalten: „Das Auftreten einer schwerwiegenden Erkrankung wurde üblicherweise wie auch die diagnostischen und therapeutischen Maßnahmen und in kurzen Abständen der Krankheitsverlauf in der Krankengeschichte dokumentiert."16

14 Ebd., S. 172.

15 von Tiedemann/Hohendorf, Methodik, S. 182-188.

16 Ebd., S. 180 
Daher wäre auch die fehlende Dokumentation vom Krankheitsverlauf und die darauf folgenden Maßnahmen ein Indiz für einen unnatürlichen Tod. ${ }^{17}$ Insgesamt kam die Studie zu dem Ergebnis, dass mindestens 850 Patient*innen (64,3\% der zwischen 1. September 1939 und 31. Juli 1945 verstorbenen Münchner Patient*innen) „infolge von Vernachlässigung, Nahrungsentzug und überdosierten Medikamenten gestorben"18 sind. Die Studienautor*innen halten aber fest, dass eine Unterscheidung zwischen natürlichem und nicht-natürlichem Tod nicht immer zweifelsfrei zu treffen sei. ${ }^{19}$

Felicitas Söhner u. a. schätzen die Zahl der zwischen 1939 und 1945 ermordeten körperlich oder geistig behinderten Menschen auf 220.000. Dazu zählen auch jene Morde, die in den "Kinderfachabteilungen" und während der "Aktion T4" passiert sind. Die Autor*innen gehen im Zuge der Untersuchung der Frage nach, ob es in der Zeit zwischen 1941 und 1944 in der Pflegeanstalt Günzburg in den Patient*innenakten Hinweise auf Patient*innentötungen gibt. Dafür verwendet wurden Personenstandsbücher, Ein- und Ausgangsbücher, Dokumente des Standesamts Günzburg und Jahresberichte der Pflegeanstalt Günzburg. Untersucht wurden 265 Patient*innenakten, wobei die tatsächliche Todesursache in den meisten Fällen nur vermutet werden kann.20

Die Studienersteller*innen definieren eine Reihe von Todesursachen, diese überschneiden sich zum Teil mit jenen von Cranach u. a.: natürlicher Tod, Opfer von Vernachlässigung, Opfer durch Medikamentenüberdosierung und kombinierte Todesursachen. Als Indizien, die gegen einen natürlichen Tod sprechen, nennen die Studienautor*innen das Fehlen einer Dokumentation des Krankheitsverlaufs, plötzlicher Eintritt des Todes, negativ-wertende Beurteilung des Patient*innenverhaltens oder typische Zeichen und Folgen einer Medikamentenüberdosierung und Hungerdiät. Vor dem Tod der Patient*innen wurde oft "zunehmende Hinfälligkeit" dokumentiert, aber nicht näher erörtert. ${ }^{21}$ Insgesamt kamen die Autor*innen der Studie zu dem Ergebnis, dass in der Pflegeanstalt Günzburg

"45 Patienten wahrscheinlich durch Hungerkost (dokumentiert durch rapide Gewichtsabnahme), Vernachlässigung, die Überdosierung von Medikamenten oder durch eine Kombination dieser Handlungen mit unmittelbarer oder mittelbarer Tötungsabsicht verstarben, d. h. getötet wurden."22

\section{Die Heil- und Pflegeanstalt Niedernhart zur Zeit des NS-Regimes}

\subsection{Die Vorgänge um Direktor Rudolf Lonauer}

Im September 1867 wurde die Heil- und Pflegeanstalt Niedernhart eröffnet. Insgesamt acht Abteilungen im Frauentrakt und sechs im Männertrakt sollten zur "Heilung von heilbaren und zur Verwahrung von gemeinschädlichen unheilbaren Geisteskranken

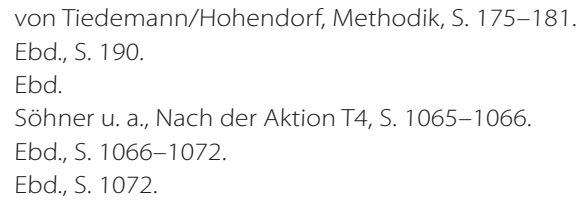


beiderlei Geschlechtes, welche nach Oberösterreich zuständig" 23 waren, zur Verfügung stehen. Einige Jahre später wurde außerdem das Schloss Gschwendt in Neuhofen an der Krems, in welches Patient*innen aus Niedernhart transferiert werden sollten, gekauft. In beiden Anstalten pflegten die Schwestern vom Orden des heiligen Vinzenz von Paul. ${ }^{24}$

1938 wurde Rudolf Lonauer Direktor der Heil- und Pflegeanstalt Niedernhart. Er leitete aber nicht nur diese, sondern ebenso die Tötungsanstalt Hartheim, in der er in den Jahren 1940 und 1941 viel Zeit verbrachte..25 Niedernhart wurde aber, so Matzek, schließlich eine Drehscheibe für dezentrale „Euthanasie". Immer wieder kamen Patient*innen nach Linz - vor allem aus den Pflegeanstalten Gschwendt, Baumgartenberg und Hardt - und wurden teilweise auch wieder in diese zurückgeschickt. ${ }^{26}$ Wann und wie genau der Leiter der Anstalt Niedernhart mit der Ermordung von Patient*innen begann ist unklar. Einig ist sich die Forschungsliteratur aber über den ungefähren Zeitraum: Heinz Faulstich hält in seinem Werk "Hungersterben in der Psychiatrie 1914-1949“ fest, dass Lonauer bereits während der "Aktion T4" Menschen mit Giftspritzen getötet haben soll. ${ }^{27}$ Dies schreibt auch Marckhgott, der sich auf die Aussage eines Pflegers bezieht, und auch Brigitte Kepplinger, merkt an, dass die Tötung von Patient*innen mit in Wasser aufgelösten Medikamenten bereits 1940 begann. ${ }^{28}$

Die Morde geschahen in vorwiegend zwei Abteilungen, die für diesen Zweck abgeschottet waren. Nach Aussage eines Pflegers wurde zunächst Abteilung VIII, dann auch Abteilung $\vee$ Lonauer direkt unterstellt. In diesen sollten „bestimmte Kranke“ untergebracht werden. ${ }^{29}$ Erstere war ursprünglich eine Männerabteilung, im März 1940 wurde diese geräumt und die Pflegelinge aus der Anstalt Hartheim untergebracht. ${ }^{30}$ Betreten durften die entsprechenden Abteilungen nur noch Lonauer selbst, sein Stellvertreter Georg Renno, Oberpfleger Karl Harrer und Oberschwester Gertrude Blanke.. ${ }^{31}$ Die Patient*innen wurden mit einer langsamen Überdosierung des Narkotikums Luminal getötet, unzureichende Nahrung und offene Fenster im Winter sollten den Prozess beschleunigen. ${ }^{32}$ Die Verantwortlichen gingen bei der Vergiftung mit Luminal nach einem von Paul Nitsche (Direkter der Heil- und Pflegeanstalten Leipzig-Dösen und Pirna-Sonnenstein sowie Gutachter der "Aktion T4") und Georg Renno ausgearbeitetem Verfahren vor. Nach diesem sollten die geschwächten Anstaltsinsass*innen mit einer eigentlich nicht tödlichen Dosis von Narkotika getötet werden. Die Opfer zeigten Läh-

23 Markus Rachbauer, Vom Verwahrungsort zur Heilanstalt? Die psychiatrische Anstalt Niedernhart 1918-1938, in: Oberösterreichisches Landesarchiv (Hrsg.), Oberösterreich 1918-1938 (4), Linz 2016, S. 66-67.

29 Christina Altenstrasser u. a., Niedernhart. Juni 1946. Ein Bericht, in: Justiz und Erinnerung (2003), Heft 8, S. 6-13, hier S. 8

30 Brigitte Kepplinger, Die Tötungsanstalt Hartheim 1940-1945, in: Brigitte Kepplinger u. a. (Hrsg.), Tötungsanstalt Hartheim, Linz 2008, S. 63-116, hier S. 70.

31 Matzek, Mordschloss, S. 180-181.

32 Ebd. 
mungs- und Erstickungserscheinungen, der Tod sollte, so Walter Schmuhl, möglichst natürlich aussehen. ${ }^{33}$

In kurzen Abständen soll Lonauer außerdem große Mengen von Luminaltabletten bestellt haben. ${ }^{34}$ Dies gab auch der Apotheker Ferdinand Glecher an. ${ }^{35}$ Dass an das Pflegepersonal tatsächlich keine Gabe von Injektionen delegiert worden war, ist, so Kepplinger, unwahrscheinlich. Alle im Zuge der gerichtlichen Aufarbeitung befragten Zeug*innen hielten aber an dieser Version fest. Im September 1943 sank die Sterberate schließlich rapide - Lonauer musste zur Waffen-SS. ${ }^{36}$ Während im Dezember 1939 1.128 Patient*innen in Niedernhart untergebracht waren, waren es im August 1943 nur mehr 323. Die restlichen Betten wurden mit Verwundeten belegt. Die letzte Tötung Lonauers fand, so Kepplingers Theorie, nach seiner Rückkehr von der Front im April 1945 statt. Wahrscheinlich wollte er die Dosierung für seinen Suizid testen - kurz vor Kriegsende töteten er und seine Frau ihre zwei Töchter und sich selbst. ${ }^{37}$

Die Vorgänge in der Anstalt wurden bereits im Juni 1945 vom interimistischen Leiter der Heil- und Pflegeanstalt Niedernhart, Dr. Wiesinger, festgehalten. In einem Schreiben an die Gesundheitsabteilung der oberösterreichischen Landeshauptmannschaft lassen sich die Vorgänge bereits klar nachzeichnen. So schreibt er, dass er den suspendierten Pfleger Leopold Lang vernommen habe. Dieser gab an, eine Verschwiegenheitserklärung unterschrieben zu haben, dessen Bruch die Todesstrafe bedeutet hätte. ${ }^{38}$ Der Pfleger zeichnet ein deutliches Bild:

"Nach diesen großen Transporten [nach Hartheim bis August 1941], von denen Steubl [ein Pfleger] nicht mehr in die Anstalt zurückkehrte, wurde zuerst Abteilung VIII, später Abteilung V von Dir. Lonauer als eigene Abteilung bestimmt, wo von ihm bestimmte Kranke unterzubringen waren. Bei männlichen Kranken machten ich und Harrer [ein Pfleger], zeitweise auch mir fremde Pfleger aus Österreich und dem Altreich Dienst, bei weiblichen eine gewisse Gertrude Blanke aus Berlin und andere mir nicht bekannte Pflegerinnen. Meine Aufgabe blieb nach wie vor die gleiche. Dir. Lonauer kam öfters und verabreichte den Kranken Injektionen, die nach 1/4 h den Tod der Patienten herbeiführten. Auch Harrer und die erwähnte Blanke verabreichten Injektionen mit dem gleichen Erfolg, besonders bei Verhinderung Dr. Lonauers. Ich musste dabei öfters assistieren, habe aber nie eine Injektion gegeben. Die Verstorbenen musste ich dann ins Leichenhaus abtragen. Anderen Kranken mussten von Dir. Lonauer bestimmte Medikamente verabfolgt werden, solch behandelte Kranke starben meist erst in einigen Tagen." ${ }^{\prime 39}$

33 Schmuhl, Rassenhygiene, S. 223.

34 Florian Schwanninger, Hartheim und Niedernhart. Zwei Stätten der NS-Euthanasie in Oberösterreich, in: Waltraud Häupl (Hrsg.), Der organisierte Massenmord an Kindern und Jugendlichen in der Ostmark 1940-1945. Gedenkdokumentation für die Opfer der NS-Euthanasie, Wien u. a. 2008, S. 161-172, hier S. 166.

35 Gabriella Hauch, Frauen. Leben. Linz. Eine Frauen- und Geschlechtergeschichte im 19. und 20. Jahrhundert, Linz 2013, S. 509.

36 Kepplinger, Krankenmord, S. 70-72; Marckhgott, „Euthanasie“, S. 175.

37 Kepplinger, Krankenmord, S. 70-72.

38 Altenstrasser u. a., Niedernhart, S. 7.

39 Ebd., S. 8. 


\subsection{Die statistische Aufarbeitung dezentraler "Euthanasie" in Niedernhart}

Gerhart Marckhgott hat eine große Menge an Daten zu Niedernhart untersucht und seine Ergebnisse bereits 1994 in einem Aufsatz festgehalten. Befasst hat er sich mit Anhaltungsakten des Amtsgerichts Linz, mit Zeug*innenaussagen, die er in den Akten des Volksgerichts Linz gefunden hatte, und mit Altakten des heutigen Neuromed-Campus. Erstere zeigen auf, dass Geisteskranke für die Dauer ihrer Krankheit entmündigt werden sollten. Ein Richter sollte in regelmäßigen Abständen die Voraussetzungen für eine Anhaltung in der Anstalt Niedernhart prüfen, doch dies war meist nicht notwendig. Viele der Patient*innen starben wenige Tage nach ihrer Ankunft. Marckhgott schließt aus dieser Beobachtung, dass die Todesursachen dieser Menschen keine natürlichen sein können. Letzteres genanntes Quellenmaterial, die Altakten des Neuromed-Campus, ist fast vollständig erhalten. In den Hauptbüchern, die Marckhgott verwendet hat, finden sich die wesentlichen Daten zu jeder Person, die in Niedernhart behandelt wurde. ${ }^{40}$ „Die Euthanasieaktionen sind hier als lange Kolonne von roten Kreuzen oft schon auf den ersten Blick zu erkennen." ${ }^{41}$ Erschwert werde die Forschung aber durch den Umstand, dass die Tötungen als natürliche Tode getarnt wurden. Daher kam es zu keiner Zerstörung der Akten - dafür hätte niemand einen Grund gesehen. ${ }^{42}$

Die Ergebnisse der Untersuchungen wurden von Marckhgott in Graphiken festgehalten. Er hat vor allem in seiner Aufzeichnung über die Transporte von und nach Niedernhart die vielen schubweisen Aufnahmen und Entlassungen deutlich gemacht. ${ }^{43}$ Mit dem Stopp der "Aktion T4" stiegen die wöchentlichen Todeszahlen sowie die Anzahl der Transporte aus den Pflegeheimen Baumgartenberg und Gschwendt nach Niedernhart. Der Höhepunkt der dezentralen Euthanasie findet sich im Frühsommer 1943, dann musste Lonauer zur Waffen-SS an die Front. Hier endet die Graphik, und Marckhgott hält fest: „Mit diesem Zeitpunkt hatte das Morden im großen Stil in Niedernhart ein Ende." 44

Marckhgott dokumentiert ebenso die monatlichen Todesfälle von 1939 bis 1943. Hier wird der Anstieg der Todesmeldungen ab August 1942 und die sehr hohe Sterblichkeitsrate im Jahr 1943 noch einmal sehr deutlich. Der Autor ist außerdem der Meinung, dass bis Sommer 1941 nur gelegentlich Patient*innen getötet wurden; den Beginn der systematischen dezentralen „Euthanasie“ in Niedernhart datiert er auf Ende 1941. Auch die durchschnittliche Aufenthaltsdauer der Verstorbenen in Niedernhart zeigt er auf. Langzeitpatient*innen waren, so Marckhgott, bereits zu einem sehr großen Teil nach Hartheim gebracht und dort ermordet worden. Nach dem Einstellen der "Aktion T4" im August 1941 fanden sich in Niedernhart fast nur mehr Patient*innen, die erst wenige Wochen zuvor aufgenommen worden waren, und 1943 betrug die durchschnittliche Aufenthaltsdauer nur mehr 38 Tage. $^{45}$

\footnotetext{
40 Marckhgott, "Euthanasie”, S. 168-169.

41 Ebd., S. 169.

42 Ebd., S. 174.

43 Ebd., S. 173-175.

44 Ebd., S. 175.

45 Ebd., S. 178-180.
} 
Insgesamt hält Gerhart Marckhgott fest, dass Lonauer nicht wahllos Patient*innen getötet hat. Vielmehr hat er sich in seinen Taten an eine Ermächtigung Hitlers von 1939 gehalten. Nach dieser sollte „unheilbar Kranken bei kritischster Beurteilung ihres Krankheitszustandes" der "Gnadentod gewährt" werden. ${ }^{46}$ Die Zahl der Toten kann letztendlich aber nicht festgesetzt werden, da nicht alle Quellen genau ausgewertet worden sind ${ }_{, \prime}$ sicher ist nach dem derzeitigen Kenntnisstand nur, daß in Niedernhart in großem Umfang von Dr. Lonauer und vermutlich einigen Helfern mehrere Hundert Patienten zwischen 1939 und 1945 getötet wurden."47

Andere Autor*innen haben sich ebenso mit einer statistischen Auswertung der Vorgänge in Niedernhart befasst, dabei aber unterschiedliche Aspekte untersucht: In ihrer Diplomarbeit hat sich Gisa Starzengruber mit Todesfällen von Kindern und Jugendlichen in Niedernhart beschäftigt und festgestellt, dass $87 \%$ der Untersuchten die Diagnose "Schwachsinn" trugen. Offizielle Todesursache war meist Lungenentzündung, Herzversagen, Tod bei epileptischem Anfall oder Darmkatarrh, und die Patient*innen blieben höchstens wenige Wochen in der Anstalt. Quellen für diese Untersuchung waren Hauptbücher, Totenscheine und Krankenakten. ${ }^{48}$

\section{Untersuchung der Krankenakten}

\subsection{Methode}

Die Untersuchung orientiert sich an der von Cranach u. a. vorgestellten Methode. Anhand der von Tiedemann u. a. vorgestellten Definition (vgl. Kapitel 1.2) wurde für diese Arbeit eine eigene Herangehensweise entwickelt. Hierbei wird die Todesursache "strukturelle Vernachlässigung" ausgeklammert. Diese war nicht nur ein Phänomen der nationalsozialistischen Zeit, sondern trat bereits im Ersten Weltkrieg schlicht aufgrund schlechter Versorgung in der gesamten Bevölkerung auf. ${ }^{49}$ Daher lautet die in dieser Arbeit verwendete Definition, angelehnt an die Forschungsgruppe für das Münchner Gedenkbuch, wie folgt: Zu dezentraler „Euthanasie“ zählt Tod infolge von bewusstem Verzicht pflegerischer und medizinischer Unterstützung, systematischem Nahrungsmittelentzug oder von bewusster Überdosierung von Medikamenten.

In der vorliegenden Arbeit wird außerdem nicht zwischen den verschiedenen Todesursachen unterschieden, sondern schlicht versucht, den Tod der Patient*innen als natürlich oder nicht-natürlich einzuordnen. Letztere Bezeichnung wird verwendet, wenn die hier aufgestellte Definition von „Euthanasie“ zutrifft, erstere, wenn die angeführte Todesursache, wie Lungenentzündung oder ein epileptischer Anfall, anhand der Vorgeschichte und der angeführten Symptome glaubwürdig erscheint. Eine weitere Unterscheidung ist aufgrund des vorhandenen Materials nicht möglich, beziehungsweise

46 von Tiedemann/Hohendorf, Methodik, S. 175-181.

47 Ebd., S. 182

48 Gisa Starzengruber, Zum Heile der Irren“, Ermordung von Kindern und Jugendlichen in der Heil- und Pflegeanstalt Niedernhart im Nationalsozialismus, Dipl. Wien 2007, zit. nach Schwanninger, Hartheim und Niedernhart, S. 167168. 
wäre sie schlicht nicht mehr treffsicher. Wie Söhner u. a. zu ihrer Forschung zur Pflegeanstalt Günzburg schreiben, liegt die

"Schwierigkeit einer sicheren historischen Bearbeitung der,dezentralen Euthanasie' [..] u. a. darin, dass die gezielte Ermordung in den Akten nicht dokumentiert wurde. Die Ermordung ist auf Grundlage des Aktenstudiums in der Regel allenfalls als höchst wahrscheinlich anzunehmen".50

Hilfreich bei der Einordnung der Akten sind die von Cranach u. a. festgelegten Kriterien. Aufgrund des vorhandenen Datenmaterials sind für diese Arbeit die Verhaltensbeschreibungen, der Pflegeaufwand, die Sprache in den Krankenakten sowie die Verlaufseintragungen der Krankengeschichte relevant. Daneben ist, wenn genannt, die Unterbringung in die Abteilung von Interesse, da die zwei Abteilungen $V$ und VIII als "Lonauers Abteilungen" gelten. ${ }^{51}$ Werden zudem die Selektionskriterien der "Aktion T4" beachtet, kann auch die Diagnose ein Indiz darstellen. Schizophrenie zum Beispiel galt als unheilbar, und hieran erkrankte Patient*innen überlebten die „Aktion T4" oft nicht.52

\subsection{Das Quellenkorpus}

Für die Untersuchung der These wurden Krankenakten aus dem Archiv des Gedenkortes Hartheim, in dem gleichzeitig die Akten der Heil- und Pflegeanstalt Niedernhart untergebracht sind, herangezogen. Insgesamt zwölf Patient*innenakten, alle aus dem Raum Vöcklabruck stammend, wurden für die Untersuchung ausgewählt. Wie bereits in der Einleitung erwähnt, ist die geringe Zahl dem begrenzten Umfang der Arbeit zuzuschreiben. Die Auswahl der einzelnen Akten erfolgte nach den räumlichen Gegebenheiten: Alle angeführten Patient*innen stehen auf unterschiedliche Weise (Geburtsort, Verwandte, etc.) in Verbindung zum Heimatort der Verfasserin. Zeitlich wurde das Sample außerdem auf Todesdaten zwischen September 1942 und Mai 1943 festgelegt, da, wie bereits erläutert, in diesem Zeitraum dezentrale „Euthanasie" sicher stattgefunden hat.

Das auf den Akten angegebene Geburtsjahr liegt zwischen 1863 und 1909. Alle Originalakten sind mithilfe ihrer Stammnummer, die mit dem ersten Aufenthalt in Niedernhart vergeben und dann immer wieder verwendet wurde, im Hartheimer Archiv zu finden. Zur Ergänzung einiger Informationen wurden aber auch teilweise die Sterbescheine der Personen herangezogen - so wurde jede der zwölf Krankenakten vom Leiter der Anstalt, Rudolf Lonauer, unterzeichnet. ${ }^{53}$

Die untersuchten Krankenakten haben alle einen ähnlichen Vordruck als Deckblatt. Dieser wurde handschriftlich oder mithilfe einer Schreibmaschine ausgefüllt und beinhaltet die wichtigsten Daten zu den Patient*innen. Vermerkt wurden:

50 Söhner u. a., Nach der Aktion T4, S. 1066.

51 Schwanninger, Hartheim und Niedernhart, S. 165-166.

52 Maike Rotzoll, Krankheit schreiben in der Psychiatrie um 1900? Diagnosen, Kranken- und Patientengeschichten von Opfern der nationalsozialistischen „Euthanasie“-Aktion „T4“, in:Yvonne Wübben/Carsten Zelle (Hrsg.), Krankheit schreiben. Aufzeichnungsverfahren in Medizin und Literatur, Göttingen 2013, S. 109-128, hier S. 111-113. 
- Vor- und Zuname ${ }^{54}$

- Tag der Aufnahme

- Tag der Entlassung

- $\quad$ Art der Entlassung

- die Stammnummer

- die Anzahl der Aufnahmen in Heil- und Pflegeanstalten

Zur Person wurde ausgefüllt:

- das Geburtsdatum

- die Konfession

- der Familienstand

- der Beruf

- der Geburtsort mit Landkreis und Gau

- die Heimatgemeinde mit Landkreis und Gau

- der ständige Wohnsitz

- der letzte Aufenthalt

Daneben bleibt auf den Deckblättern auch Platz für:

- die Zulässigkeit der Anhaltung

- die Kuratelsbehörde

- den Kurator

- die Postanschrift der Angehörigen

Diese Punkte wurden aber nicht immer ausgefüllt. Zur Diagnostik wird vermerkt:

- die Diagnose des Jahresberichtes

- die klinische Diagnose (angegeben mithilfe des Würzburger Schlüssels)

- die Ätiologie (Allgemeine und Herediät)

- frühere Anstaltsbehandlungen

- der Beginn der Erkrankung

- $\quad$ kam vom Militär aus der Untersuchung oder Strafhaft

In einem Feld rechts unten befindet sich auf den Vordrucken außerdem ein kleines Kästchen, dessen leerer Platz mit den Worten „Zwilling:" und „Erbärztliche Maßnahme:"

54 In dieser Arbeit wird der Zuname mit dem ersten Buchstaben abgekürzt. In den letzten Jahren gibt es vermehrt Debatten zur Namensnennung von Opfern: Befürworter*innen wollen den Opfern ein Stück Identität wiedergeben, Angehörige hingegen wollen die Namen oft nicht lesen. Eine Anonymisierung wird in diesem Fall von der Dokumentationsstelle Hartheim gewünscht. 
überschrieben ist. ${ }^{55}$ Dieser ist aber in allen Akten leer und fehlt in den älteren Vordrucken (zum Beispiel in jenem von Karl K., 2. Mai 1939) ${ }^{56}$ völlig.

Gefüllt sind die Akten der sieben Patienten und fünf Patientinnen sehr unterschiedlich: In manchen finden sich sehr viele Informationen, auch zu früheren Erkrankungen und Aufenthalten; ebenso gibt es eine umfassende Anamnese, wie zum Beispiel in der Akte von Maria B., ${ }^{57}$ Entmündigungsverfahren wurden ebenso festgehalten, wie im Fall von Maria K. ${ }^{58}$ Andere Akten sind wiederum nur wenig gefüllt. So lassen sich zu Franziska E. und Johanna H. nur das Deckblatt finden. ${ }^{59}$ Hieraus Schlussfolgerungen zu ziehen ist nur schwer und verbunden mit einigen Spekulationen möglich. Die folgende Tabelle soll einen ersten Überblick über die untersuchten Patient*innenakten, sortiert nach der Stammnummer, geben:

\begin{tabular}{|c|c|c|c|c|c|}
\hline Name & Stammnr. & Geburtsdatum & $\begin{array}{l}\text { Datum der } \\
\text { Aufnahme }\end{array}$ & Sterbedatum & $\begin{array}{c}\text { Todesursache laut } \\
\text { Krankenakte }\end{array}$ \\
\hline Maria K. & 9.834 & 7. Mai 1883 & 10. April 1941 & $\begin{array}{l}\text { 18. Dezember } \\
1941\end{array}$ & Herzmuskelentartung \\
\hline Ludwig G. & 10.609 & 19. Juli 1885 & 6. Mai 1942 & 9. Juli 1942 & Apoplexia cerebri \\
\hline Maria B. & 12.730 & 6. April 1883 & 4. April 1943 & 8. Mai 1943 & Herzmuskelentartung \\
\hline Karl K. & 15.316 & $\begin{array}{l}\text { 2. Dezember } \\
1919\end{array}$ & 2. Mai 1939 & $\begin{array}{l}\text { 28. Oktober } \\
1941\end{array}$ & $\begin{array}{l}\text { laut Sterbeschein: } \\
\text { Lungenentzündung }\end{array}$ \\
\hline Karl H. & 15.518 & $\begin{array}{l}\text { 28. September } \\
1900\end{array}$ & $\begin{array}{l}\text { 18. November } \\
1939\end{array}$ & $\begin{array}{l}\text { 18. September } \\
1941\end{array}$ & Herzmuskelentartung \\
\hline Josef B. & 16.240 & 21. August 1902 & $\begin{array}{l}\text { 19. Jänner } \\
1942\end{array}$ & $\begin{array}{l}\text { 29. Jänner } \\
1942\end{array}$ & epileptischer Anfall \\
\hline $\begin{array}{c}\text { Günther } \\
\text { K. }\end{array}$ & 16.266 & 3. August 1863 & $\begin{array}{l}\text { 9. Februar } \\
1942\end{array}$ & $\begin{array}{l}\text { 13. Februar } \\
1942\end{array}$ & Kachexie \\
\hline $\begin{array}{c}\text { Katharina } \\
\mathrm{H} .\end{array}$ & 16.334 & 8. März 1885 & 13. März 1942 & 16. März 1942 & Apoplexia cerebri \\
\hline Franz A. & 16.600 & $\begin{array}{l}\text { 10. Februar } \\
1909\end{array}$ & 25. Juli 1942 & $\begin{array}{l}\text { 28. August } \\
1942\end{array}$ & Lungenentzündung \\
\hline $\begin{array}{c}\text { Franziska } \\
\text { E. }\end{array}$ & 16.613 & 12. März 1874 & 27. Juli 1942 & $\begin{array}{l}\text { 25. August } \\
1942\end{array}$ & Altersschwäche \\
\hline Josef S. & 16.895 & $\begin{array}{l}\text { 12. Februar } \\
1870\end{array}$ & $\begin{array}{l}\text { 13. Jänner } \\
1943\end{array}$ & $\begin{array}{l}\text { 26. Jänner } \\
1943\end{array}$ & Nephrosclerosis \\
\hline $\begin{array}{c}\text { Johanna } \\
\text { H. }\end{array}$ & 17.149 & $\begin{array}{l}\text { 12. Dezember } \\
1904\end{array}$ & 4. Mai 1943 & 6. Mai 1943 & Knochenkaries \\
\hline
\end{tabular}

55 Ein Beispiel: Krankenakt von Maria B., 8.4.1943. Opferdatenbank der Dokumentationsstelle Hartheim des Oö. Landesarchivs, Stammnummer 12.730.

56 Krankenakt von Karl K., 6.9.1939. Opferdatenbank der Dokumentationsstelle Hartheim des Oö. Landesarchivs, Stammnummer 15.316.

57 Krankenakt von Maria B., 8.4.1943. Opferdatenbank der Dokumentationsstelle Hartheim des Oö. Landesarchivs, Stammnummer 12.730 .

58 Krankenakt von Maria K., 18.12.1941. Opferdatenbank der Dokumentationsstelle Hartheim des Oö. Landesarchivs, Stammnummer 9.834.

59 Krankenakt von Franziska E., 25.8.1942. Opferdatenbank der Dokumentationsstelle Hartheim des Oö. Landesarchivs, Stammnummer 16.613 und Krankenakt von Johanna H., 6.5.1943. Opferdatenbank der Dokumentationsstelle Hartheim des Oö. Landesarchivs, Stammnummer 17.149.

60 Dokumentationsstelle Hartheim, Sterbescheine Anstalt Niedernhart 1938-1945 - digitales Verzeichnis. 


\subsection{Bewertung der einzelnen Krankenakten}

Es folgt eine Bewertung der Krankenakten nach den angegebenen Kriterien (vgl. Kapitel 3.1). Genannt werden die wichtigsten Aspekte, bevor eine Einschätzung gemacht wird.

\section{Maria K.}

Maria K. ist am 18. Dezember 1941 im 58. Lebensjahr in Niedernhart verstorben. Sie war bereits das zweite Mal in der Heil- und Pflegeanstalt. Der erste Aufenthalt dauerte drei Monate im Jahr 1920. Als Diagnose wurde "Schizophrenie" auf der Akte vermerkt. Am 20. August 1941 wurde Maria voll entmündigt, Kurator wurde ihr Gatte Franz K. Die Patientin wurde bei ihrer Einlieferung am 10. April 1941 als "geistig recht zerfahren" und „über und über mit Ungeziefer bedeckt” beschrieben. Der Pflegeaufwand war folglich zunächst groß, außer der „Säuberung von Ungeziefer", deren Dokumentation ca. eine halbe Seite einnimmt, wurden aber bis zu ihrem Tod einige Monate später keine weiteren Aufzeichnungen gemacht. Die Sprache ist neutral gehalten. Die Diagnose und die fehlenden Verlaufseintragungen können ein Indiz für einen nicht-natürlichen Tod sein. Letztendlich kann aber keine genaue Zuordnung getroffen werden, da die Todesursache, "Herzmuskelentartung",61 ein heute nicht mehr verwendeter Begriff, sowohl langsam auftretende Herzschwäche, als auch akutes Herzversagen bedeuten kann. ${ }^{62}$ Außerdem ist anzumerken, dass die "Aktion T4" noch ein paar Monate andauerte, als Maria K. in die Anstalt kam.

\section{Ludwig G.}

Ludwig G. ist am 9. Juli 1942 im 57. Lebensjahr in Niedernhart verstorben. Ein früherer Aufenthalt im Jahr 1923 dauerte vier Monate und von 23. Jänner bis 12 . März 1942 wurde er erneut in Niedernhart behandelt. Als Diagnose wurde "Schizophrenie" (Nr. 14), "Paranoia querulaturo" und "Alkoholismus" auf der Akte vermerkt. Eingeliefert wurde er als Gefangener "wegen Hochverratssache“, wie aus einem Transportschein hervorgeht, der ebenfalls dem Krankenakt beiliegt. In einem Gutachten, geschrieben von Lonauer am 27. Februar 1942, steht ohne weitere Begründung, dass er Kontakt zu Kommunist*innen hatte. ${ }^{63}$ Aufmerken lässt hier der Umstand, dass sich ein Arzt, noch dazu der Direktor von Niedernhart, mit der politischen Einstellung eines seiner Patienten befasst hat.

Bei der Aufnahme wurde er als "gemeingefährlicher Geisteskranker“ laut gerichtsärztlichem Gutachten beschrieben. Er sei außerdem, so die Eintragungen in den Krankenakt, ,/ästig und zudringlich". In den letzten zwanzig Tagen seines Aufenthaltes wurden

61 Krankenakt von Maria K., 18.12.1941. Opferdatenbank der Dokumentationsstelle Hartheim des Ö. Landesarchivs, Stammnummer 9.834.

62 Richard Geigel, Lehrbuch der Herzkrankheiten, München-Wiesbaden 1920, S. 245-147.

63 ganzer Absatz: Krankenakt von Ludwig G., 9.7.1942. Opferdatenbank der Dokumentationsstelle Hartheim des Oö. Landesarchivs, Stammnummer 10.609. 
keine Eintragungen mehr gemacht. Ebenso ist in der Akte ein Brief der Ehefrau enthalten, die das Verhalten ihres Mannes beschreibt und schlussfolgert, er könne nicht normal sein. Ludwig G. war in Abteilung $\vee$ untergebracht. All dies sind Indizien, die auf einen unnatürlichen Tod hindeuten. Ein weitere Hinweis ist hierbei noch ein Brief aus dem Jahr 1954 vom damaligen Direktor von Niedernhart. Diese Mitteilung wurde ohne weiteren Zusammenhang in die Akte eingelegt: „Aus einem Zeichen auf der letzten Krankengeschichte ist zu entnehmen, daß [G.] Ludwig der sogenannten, Euthanasie' zum Opfer gefallen ist. Die angegebene Todesursache - Apoplexia (Herzstillstand) dürfte fingiert sein [...]." ${ }^{4}$ Welches Zeichen auf dem Krankenakt der Direktor als Beweis für "Euthanasie" angesehen hat, lässt sich allerdings nicht mehr nachvollziehen, da im Vergleich zu den anderen Akten keines zu passen scheint. Die Todesursache von Ludwig G. kann aufgrund der hier angeführten Indizien als nicht-natürlich angenommen werden.

\section{Maria B.}

Maria B. ist am 8. Mai 1943 im 60. Lebensjahr in Niedernhart verstorben. Insgesamt sind in ihrer Akte vier Aufenthalte in Anstalten verzeichnet, die letzten beiden in Niedernhart werden mit der Diagnose 14 nach dem Würzburger Schlüssel, „Schizophrenie“, begründet. Dazwischen war sie in der Fürsorgeanstalt Baumgartenberg. ${ }^{65}$ Aus den Akten lässt sich schließen, dass Maria wahrscheinlich eine Langzeitpatientin war, um die sich die Geschwister oder auch verschiedene Einrichtungen gekümmert haben. Die lange Pflegebedürftigkeit und die Diagnose sprechen für eine Tötung durch den Direktor oder das Pflegepersonal. Hier aber lassen sich keine Aussagen treffen, da von Baumgartenberg keine Aufzeichnungen vorhanden sind - auch vom letzten Aufenthalt in Niedernhart nicht. Daher kann über den Gesundheitszustand der Maria B. keine Aussage gemacht werden. Die Todesursache („,Herzmuskelentartung "66) ist für das Alter von Maria B nicht ungewöhnlich, die Dokumentation des Krankheitsverlaufs, Medikation oder sonstiger Maßnahmen fehlt aber. Insgesamt kann hier von einer wahrscheinlich nicht-natürlichen Todesursache ausgegangen werden.

\section{KarlK.}

Der Krankenakt von Karl K. ist unvollständig, laut Verzeichnis der Sterbescheine ist er am 28. Oktober 1941 im 22. Lebensjahr an „Lungenentzündung" verstorben. Da zum letzten Aufenthalt in Niedernhart keine Aufzeichnungen vorhanden sind, ${ }^{67}$ lässt sich keine Aussage über die Todesursache treffen.

\footnotetext{
64 Krankenakt von Ludwig G., 9.7.1942. Opferdatenbank der Dokumentationsstelle Hartheim des Oö. Landesarchivs, Stammnummer 10.609 .

65 Krankenakt von Maria B., 8.4.1943. Opferdatenbank der Dokumentationsstelle Hartheim des Oö. Landesarchivs, Stammnummer 12.730 .

66 Ebd

67 Krankenakt von Karl K., 6.9.1939. Opferdatenbank der Dokumentationsstelle Hartheim des Oö. Landesarchivs, Stammnummer 15.316 .
} 
Karl H.

Karl H. ist am 18. September 1941 im 41. Lebensjahr in Niedernhart verstorben. Er wurde als "geistesgestörter Angestellter" in die Heil- und Pflegeanstalt Niedernhart überstellt. Hier verbrachte er über zwei Jahre, bis er schließlich mit 41 Jahren an „Herzmuskelentartung" starb. Krankheitsverlauf findet sich keiner in der Akte, die Todesursache wurde außerdem gemeinsam mit der Diagnose ("Schizophrenie im Endzustand") eingetragen, wie an Tinte und Handschrift deutlich wird. Er sei „faselig" gewesen und hätte Pfleger beschimpft, in der letzten Eintragung eine Woche vor seinem Tod wurde vermerkt, dass Karl $\mathrm{H}$. in Abteilung VIII verlegt werden solle. ${ }^{68}$ Diese Punkte lassen den Schluss zu, dass die Todesursache am Krankenakt des Patienten fingiert wurde.

Josef $B$.

Josef B. ist am 19. Jänner 1942 im 41. Lebensjahr in Niedernhart verstorben. Laut seinem Krankenakt litt er seit seinem 15. oder 16. Lebensjahr an „Epilepsie infolge eines Sturzes". Er hätte sich verwirrt und störend gezeigt, bereits nach einigen Tagen wurde er „Wegen Platzmangel” auf Abteilung VIII gebracht, dort starb er an einem epileptischen Anfall. ${ }^{69}$ Aufgrund der Angabe der Abteilung kann die Todesursache als nicht-natürlich angenommen werden.

\section{Günther K.}

Günther K. ist am 13. Februar 1942 im 79. Lebensjahr in Niedernhart verstorben. Er verbrachte nur fünf Tage dort, die Todesursache "Kachexie“ passt zu dem Notierten. Die Diagnose lautete „senile Form psychischer Störung des höheren Lebensalters"70 Es kann angenommen werden, dass Günther K. tatsächlich an einer natürlichen Todesursache starb.

\section{Katharina $\mathrm{H}$.}

Katharina H. ist am 16. März. 1942 im 58. Lebensjahr in Niedernhart verstorben. Sie war nur vier Tage dort, bevor sie laut Deckblatt an einem „Schlaganfall“ starb. Im Krankheitsverlauf wird "Marasmus bei Herzschwäche (fortschreitender Verfall)" als Todesursache angeführt. Aufgrund der wenigen Informationen lassen sich aber keine Aussagen zur Todesursache treffen. ${ }^{71}$

68 Krankenakt von Karl H., 18.9.1941. Opferdatenbank der Dokumentationsstelle Hartheim des Oö. Landesarchivs, Stammnummer 15.518.

69 Krankenakt von Josef B., 29.1.1942. Opferdatenbank der Dokumentationsstelle Hartheim des Oö. Landesarchivs, Stammnummer 16.240 .

70 Krankenakt von Günther K., 13.2.1942. Opferdatenbank der Dokumentationsstelle Hartheim des Oö. Landesarchivs, Stammnummer 16266.

71 Krankenakt von Katharina H., 16.3.1942. Opferdatenbank der Dokumentationsstelle Hartheim des Oö. Landesarchivs, Stammnummer 16.334 . 
Franz A.

Franz A. ist am 28. August 1942 im 34. Lebensjahr in Niedernhart an „Lungenentzündung" verstorben. Einen Monat war der Patient in der Heil- und Pflegeanstalt. Aus Verlaufseintragungen geht hervor, dass Franz an „Verkrüppelung der Arme und Füsse“ litt, außerdem komme er aus einer "asozialen Familie". Im Zuge der Anamnese wurde festgestellt, dass der Patient einen „ausgesprochen schwachsinnigen“ Eindruck machte. In einem Schreiben von 1954, ähnlich jenem aus Ludwig Gs. Akte, unterzeichnet vom damaligen Direktor, wird vermerkt: „Vermutlich wurde er euthanasiert."72 Die Durchsicht der Akte bestätigt den Eindruck.

\section{Franziska $E$.}

Franziska E. ist am 15. August 1942 im 68. Lebensjahr in Niedernhart an „Altersschwäche" verstorben. Sie hat einen Monat dort verbracht. Bei der Angabe der Todesursache scheint es, als wäre diese noch einmal ausgebessert worden, unter dem Begriff "Altersschwäche" finden sich andere Buchstaben, die allerdings nicht mehr lesbar sind.73 Insgesamt kann zu diesem Krankenakt aber keine Aussage getroffen werden, da Verhaltensbeschreibungen, Hinweise zum Pflegeaufwand, die Sprache über die Patientin, Verlaufseintragungen zur Krankheit und die Abteilung schlicht fehlen.

JosefS.

Josef S. ist am 26. Jänner 1943 im 73. Lebensjahr in Niedernhart an "Nephrosklerose”, einer Nierenkrankheit, verstorben. Der Krankheitsverlauf ist ausführlich dokumentiert, auch Therapiemaßnahmen, die bereits im Allgemeinen Krankenhaus in Linz durchgeführt worden waren, wurden festgehalten. ${ }^{74}$ Die angeführte Todesursache scheint zu stimmen, es gibt keine Hinweise auf Fremdeinwirken.

\section{JohannaH.}

Johanna H. ist am 6. Mai 1943 im 40. Lebensjahr in Niedernhart am „Knochencaries" verstorben. Die Todesursache passt zur Diagnose: „angeborener Schwachsinn (Taubstumm)."75 Ihr Krankenakt besteht nur aus dem Deckblatt, auf diesem wird der „Beruf" „Fürsorgepflegling" angegeben. ${ }^{76}$ Die Patientin war sehr jung und die Tatsache, dass sie Langzeitpatientin war, als auch der kurze Aufenthalt in Niedernhart - wäre sie

72 Krankenakt von Franz A., 28.8.1942. Opferdatenbank der Dokumentationsstelle Hartheim des Oö. Landesarchivs, Stammnummer 16.600.

73 Krankenakt von Franziska E., 25.8.1942. Opferdatenbank der Dokumentationsstelle Hartheim des Oö. Landesarchivs, Stammnummer 16.613

74 Krankenakt von Josef S., 26.1.1943. Opferdatenbank der Dokumentationsstelle Hartheim des Oö. Landesarchivs, Stammnummer 16.895.

75 S. Bisdas u. a., Ohr, in: Facharztwissen HNO-Heilkunde. Differenzierte Diagnostik und Therapie, Heidelberg 2009, S. 139-289, hier S. 235-236.

76 Krankenakt von Johanna H., 6.5.1943. Opferdatenbank der Dokumentationsstelle Hartheim des Oö. Landesarchivs, Stammnummer 17.149 . 
hierhin von Baumgartenberg gekommen, weil sie sehr krank war und besserer Pflege bedurfte, hätte es Aufzeichnungen geben müssen - lassen den Schluss zu, dass Franziska E. wahrscheinlich an keiner natürlichen Todesursache starb.

\subsection{Ergebnisse}

Insgesamt kann also festgehalten werden, dass aus keinem Krankenakt explizit eine Tötung von Patient*innen hervorgeht, allerdings lassen sich Indizien finden, die gegen eine natürliche Todesursache sprechen. Bei insgesamt sechs Patient*innen kann von einer wahrscheinlich nicht-natürlichen Todesursache gesprochen werden. Diese sind Maria B., Karl H., Johanna H., Ludwig G., Josef B. und Franz A. Aus zwei Krankenakten lässt sich schließen, dass die Todesursache tatsächlich stimmt: In den Fällen von Günther K. und Josef S. kann Fremdverschulden nahezu ausgeschlossen werden. Die restlichen Krankenakten sind aus verschiedenen Gründen schwer zu beurteilen und konnten daher keiner der beiden festgelegten Kategorien zugeordnet werden. Gründe dafür sind fehlendes Material (Franziska E., Karl K. und Katharina H.) oder widersprüchliche Indizien (Maria K.).

\section{Fazit}

Letztendlich kann in keiner der untersuchten Patient*innenakten „Euthanasie" definitiv nachgewiesen werden, es gibt aber durchaus Fälle, in denen die Verdachtsmomente größer sind. Erschwert wird die Untersuchung im Vergleich zur Gedenkdokumentation der Münchner Patient*innen von Eglfing-Haar durch den geringen Umfang des zur Verfügung stehenden Materials. Es fehlen Gewichtskurven, Temperaturtabellen und in manchen Fällen sogar die Anamnese. Sektionsberichte standen im Gegensatz zur Münchner Forschungsgruppe ebenfalls nicht zur Verfügung und Aufzeichnungen zur Medikation oder pathologische Befunde wurden ebenfalls nicht aufgefunden. Über den Grund für die Lückenhaftigkeit der Krankenakten - wie bereits erwähnt fehlen auch häufig Aufzeichnungen zum Krankheitsverlauf - kann heute nur spekuliert werden: Geschah dies aus Personalmangel, Gleichgültigkeit gegenüber den Patient*innen oder der Tatsache, dass das Personal ohnehin vorhatte, die Person zu töten? Diese Frage kann nicht beantwortet werden, sollte aber gerade bei der Untersuchung von Krankenakten immer mitbedacht werden, die Gründe dafür können vielfältig sein.

Dass in der Heil- und Pflegeanstalt Niedernhart Patient*innen durch Luminalspritzen getötet worden sind, wird, wie in Kapitel 2 angeführt, in der Literatur nicht bestritten. Die Arbeiten stützen sich auf Gerichtsprotokolle oder statistische Auswertungen. Dass in den Krankenakten so wenig nachgewiesen werden kann, ist sicherlich auch auf das angewendete Luminalschema zurückzuführen. Gerade dieses sollte Symptome hervorrufen, die einer natürlichen Todesursache ähnlich waren. Nicht nachvollziehbar ist außerdem, welche Auswirkungen die - mutwillige oder durch den Krieg bedingte schlechte Versorgung auf die Gesundheit der Patient*innen hatte. 
Ausgangspunkt der Arbeit war die Forschungsfrage: Kann in der Heil-und Pflegeanstalt Niedernhart anhand von Krankenakten dezentrale „Euthanasie“ zur Zeit des NS-Regimes rekonstruiert werden? Die Frage ist nicht mit einem eindeutigen "Ja" oder "Nein" zu beantworten, vielmehr sollte die These ergänzt werden. So könnte die Antwort auf die Forschungsfrage lauten: Die Ermordung von Patient*innen durch Narkotika, Nahrungsentzug, mangelndes Heizen oder Vorenthaltung wichtiger Medikamente kann anhand ausgewählter Akten aus der Heil- und Pflegeanstalt Niedernhart aufgrund von Indizien - beispielsweise den Pflegeaufwand, die Sprache über die Patient*innen, Verhaltensbeschreibungen, die behandelnde Abteilung oder fehlende Verlaufseintragungen zu Krankheiten - vermutet werden, dies wird aber durch das Fehlen wichtiger Eintragungen, wie zum Beispiel der Medikation, erschwert.

\section{Literatur}

Altenstrasser, Christina u. a., Niedernhart. Juni 1946. Ein Bericht, in: Justiz und Erinnerung (2003), Heft 8, S. 6-13.

Bisdas, S. u. a., Ohr, in: Facharztwissen HNO-Heilkunde. Differenzierte Diagnostik und Therapie, Heidelberg 2009, S. 139-289.

von Cranach, Michael u. a. (Hrsg.), Gedenkbuch für die Münchner Opfer der nationalsozialistischen „Euthanasie“-Morde, Göttingen 2018.

Faulstich, Heinz, Hungersterben in der Psychiatrie. Mit einer Topographie der NS-Psychiatrie, Freiburg im Breisgau 1998.

Gabriel, Eberhard, NS-Euthanasie in Österreich von 1938 bis 1945. Die Ereignisse - eine Rekapitulation, in: psychopraxis.neuropraxis 19 (2016), Heft 1, S. 21-24.

Geigel, Richard, Lehrbuch der Herzkrankheiten, München-Wiesbaden 1920.

Hauch, Gabriella, Frauen. Leben. Linz. Eine Frauen- und Geschlechtergeschichte im 19. und 20. Jahrhundert, Linz 2013.

Kepplinger, Brigitte, Regionalisierter Krankenmord. Voraussetzungen und Strukturen der nationalsozialistischen Patiententötung außerhalb der zentral gesteuerten Programme, in: Bertrand Perz u. a. (Hrsg.), Schlussbericht der Kommission zur Untersuchung der Vorgänge um den Anstaltsfriedhof des Psychiatrischen Krankenhauses in Hall in Tirol in den Jahren 1942 bis 1945, Innsbruck 2014, S. 49-82.

Dies., Die Tötungsanstalt Hartheim 1940-1945, in: Brigitte Kepplinger u. a. (Hrsg.), Tötungsanstalt Hartheim, Linz 2008, S. 63-116.

Marckhgott, Gerhart, Euthanasie in Oberdonau, in: Zeitgeschichte 21 (1994), Heft 5, S. 165-182.

Matzek, Tom, Das Mordschloss. Auf den Spuren von NS-Verbrechen in Schloss Hartheim, Wien 2003. 
Neugebauer, Wolfgang, NS-Euthanasieaktionen in Österreich. Ein Überblick, in: Bertrand Perz u. a. (Hrsg.), Schlussbericht der Kommission zur Untersuchung der Vorgänge um den Anstaltsfriedhof des Psychiatrischen Krankenhauses in Hall in Tirol in den Jahren 1942 bis 1945, Innsbruck 2014, S. 35-48.

Ders., Die „Aktion T4", in: Brigitte Kepplinger u. a. (Hrsg.), Tötungsanstalt Hartheim, Linz 2008, S. 17-34.

Rachbauer, Markus, Vom Verwahrungsort zur Heilanstalt? Die psychiatrische Anstalt Niedernhart 1918-1938, in: Oberösterreichisches Landesarchiv (Hrsg.), Oberösterreich 1918-1938 (4), Linz 2016.

Rotzoll, Maike, Krankheit schreiben in der Psychiatrie um 1900? Diagnosen, Krankenund Patientengeschichten von Opfern der nationalsozialistischen „Euthanasie“-Aktion „T4", in: Yvonne Wübben/Carsten Zelle (Hrsg.), Krankheit schreiben. Aufzeichnungsverfahren in Medizin und Literatur, Göttingen 2013, S. 109-128.

Schmuhl, Hans-Walter, Rassenhygiene, Nationalsozialismus, Euthanasie. Von der Verhütung zur Vernichtung "lebensunwerten Lebens" 1890-1945 (Kritische Studien zur Geschichtswissenschaft 75), Göttingen 1987.

Schwanninger, Florian, Hartheim und Niedernhart. Zwei Stätten der NS-Euthanasie in Oberösterreich, in: Waltraud Häupl (Hrsg.), Der organisierte Massenmord an Kindern und Jugendlichen in der Ostmark 1940-1945. Gedenkdokumentation für die Opfer der NS-Euthanasie, Wien u. a. 2008, S. 161-172.

Söhner, Felicitas u. a., Nach der Aktion T4. "regionalisierte Euthanasie” in der Heil- und Pflegeanstalt Günzburg, in: Nervenarzt 88 (2017), Heft 9, S. 1065-1073.

Süß, Winfried, Der „Volkskörper“ im Krieg. Gesundheitspolitik, Gesundheitsverhältnisse und Krankenmord im nationalsozialistischen Deutschland 1939-1945, München 2003.

von Tiedemann, Sibylle/Hohendorf, Geritt, Methodik. Die Ermittlung der Opfer der dezentralen „Euthanasie“, in: Michael von Cranach u. a. (Hrsg.), Gedenkbuch für die Münchner Opfer der nationalsozialistischen „Euthanasie“-Morde, Göttingen 2018, S. 169-192.

\section{Quellen}

Dokumentationsstelle Hartheim, Sterbescheine Anstalt Niedernhart 1938-1945 - digitales Verzeichnis.

Krankenakten aus der Opferdatenbank der Dokumentationsstelle Hartheim des Oö. Landesarchivs, Stammnummern 16.600, 16.613, 16.266, 17.149, 16.240, 16.895, 15.518, $15.316,16.334,10.609,12.730,9.834$.

Anna Kirchgatterer ist Studentin des Lehramtsstudiums in den Fächern Deutsch und Geschichte und der Geschichtswissenschaften an der Universität Innsbruck. anna.kirchgatterer@student.uibk.ac.at 


\section{Zitation dieses Beitrages}

Anna Kirchgatterer, Dezentrale "Euthanasie” in der Heil- und Pflegeanstalt Niedernhart. Untersuchung am Beispiel ausgewählter Krankenakten, in: historia. scribere 12 (2020), S. 59-79, [http://historia.scribere.at], eingesehen 25.5.2020 (=aktuelles Datum).

(C) Creative Commons Licences 3.0 Österreich unter Wahrung der Urheberrechte der Autorlnnen. 
